PROC. OF JSCE,

No. 290 , OCT. 1979

【Technical Note】

\title{
GENERAL FORMS OF GALERKIN VECTOR IN CYLINDRICAL COORDINATES
}

\author{
By Hiroyoshi HIRAI* and Masao SATAKE**
}

\section{INTRODUCTION}

The various forms of solution of Navier's equation expressed in terms of potentials have been proposed for the analysis of three-dimensional elastic problems. In the case of cartesian coordinates, many solutions are proposed by Neuber ${ }^{1)}$, Papkovich $^{1)}$, Boussinesq ${ }^{2)}$, Galerkin ${ }^{1)}$ and so on. In the case of cylindrical coordinates, there is the solution by Love or Michell ${ }^{3)}$ for axisymmetric problems and its generalization was studied by Hasegawa $^{4}$. Further, Muki ${ }^{5)}$ proposed a solution for asymmetric problems and the completeness of Muki's solution was discussed by $\mathrm{Hata}^{6}$.

The above solutions in cylindrical coordinates are considered to be closely related with the solution expressed in terms of Galerkin vector. From this viewpoint, the general forms of Galerkin vector in cylindrical coordinates are proposed and their application to an asymmetric problem is shown in this paper.

\section{GALERKIN VECTOR IN CYLINDRICAL COORDINATES}

If the body force is absent, Navier's equation is expressed as

$$
\nabla^{2} \boldsymbol{u}+\frac{1}{1-2 \nu} \nabla(\nabla \cdot u)=0,
$$

where $\boldsymbol{u}$ is the displacement vector and $\nu$ is Poisson's ratio. Galerkin introduced a solution of Eq. (1) with the form

$$
\begin{aligned}
& 2 \mu \boldsymbol{u}=2(1-\nu) \nabla^{2} \boldsymbol{G}-\nabla(\nabla \cdot \boldsymbol{G}), \\
& \nabla^{4} \boldsymbol{G}=\mathbf{0}, \ldots \ldots \ldots \ldots \ldots \ldots \ldots \ldots \ldots \ldots \ldots \ldots \ldots \ldots
\end{aligned}
$$

where $\boldsymbol{G}$ is called the Galerkin vector and $\mu$ denotes Lamé's modulus. In the following, gen-

* M. Eng. Postgraduate of Civil Engineering, Tohoku University

** Dr. Eng. Professor of Civil Engineering, Tohoku University eral expressions of Galerkin vector in cylindrical coordinates are investigated. As Eq. (3) is considered to be a biharmonic equation, $\boldsymbol{G}$ is to be a biharmonic vector. Putting

$$
\begin{aligned}
& \nabla^{2} \boldsymbol{G}=\boldsymbol{g}, \\
& \nabla^{2} \boldsymbol{g}=\mathbf{0}, \\
& \boldsymbol{G}=\left(G_{r}, G_{\theta}, G_{z}\right), \\
& \boldsymbol{g}=\left(g_{r}, g_{\theta}, g_{z}\right),
\end{aligned}
$$

Eq. (5) is expressed in the form

$$
\begin{aligned}
\left(\nabla^{2}-\frac{1}{r^{2}}\right) g_{r}-\frac{2}{r^{2}} \frac{\partial}{\partial \theta} g_{\theta} & =0, \\
\frac{2}{r^{2}} \frac{\partial}{\partial \theta} g_{r}+\left(\nabla^{2}-\frac{1}{r^{2}}\right) g_{\theta} & =0, \\
\nabla^{2} g_{z} & =0,
\end{aligned}
$$

where

$$
\nabla^{2}=\frac{\partial^{2}}{\partial r^{2}}+\frac{1}{r}-\frac{\partial}{\partial r}+\frac{1}{r^{2}} \frac{\partial^{2}}{\partial \theta^{2}}+\frac{\partial^{2}}{\partial z^{2}} .
$$

Referring the formulas for derivatives and recurrence relations in the Bessel functions, it is easy to show that the solutions of Eq. (8) can be given from combination of $g_{r}, g_{\theta}$ and $g_{z}$ listed in Table 1. On the other hand, Eq. (4) is expressed in the form

$$
\left.\begin{array}{rl}
\left(\nabla^{2}-\frac{1}{r^{2}}\right) G_{r}-\frac{2}{r^{2}} \frac{\partial}{\partial \theta} G_{\theta} & =g_{r}, \\
\frac{2}{r^{2}} \frac{\partial}{\partial \theta} G_{r}+\left(\nabla^{2}-\frac{1}{r^{2}}\right) G_{\theta} & =g_{\theta}, \\
\nabla^{2} G_{z} & =g_{z} .
\end{array}\right\}
$$

Substituting the solutions of Eq. (8) into the right-hand side of Eq. (10) and using the theorem of Almansi ${ }^{7)}$ in cylindrical coordinates, the solutions of Eq. (10) can be given from combination of $G_{r}, G_{\theta}$ and $G_{z}$ listed in Table 1. In Table 1, $J_{k}(\alpha r)$ and $Y_{k}(\alpha r)$ are the Bessel functions of the first kind and the second kind of order $k$ respectively, and $I_{k}(\beta r)$ and $K_{k}(\beta r)$ are the modified Bessel functions of the first and the second kind of order $k$ respectively, where $\alpha$ and $\beta$ are constants. 
Table 1 General forms of biharmonic vector in cylindrical coordinates.

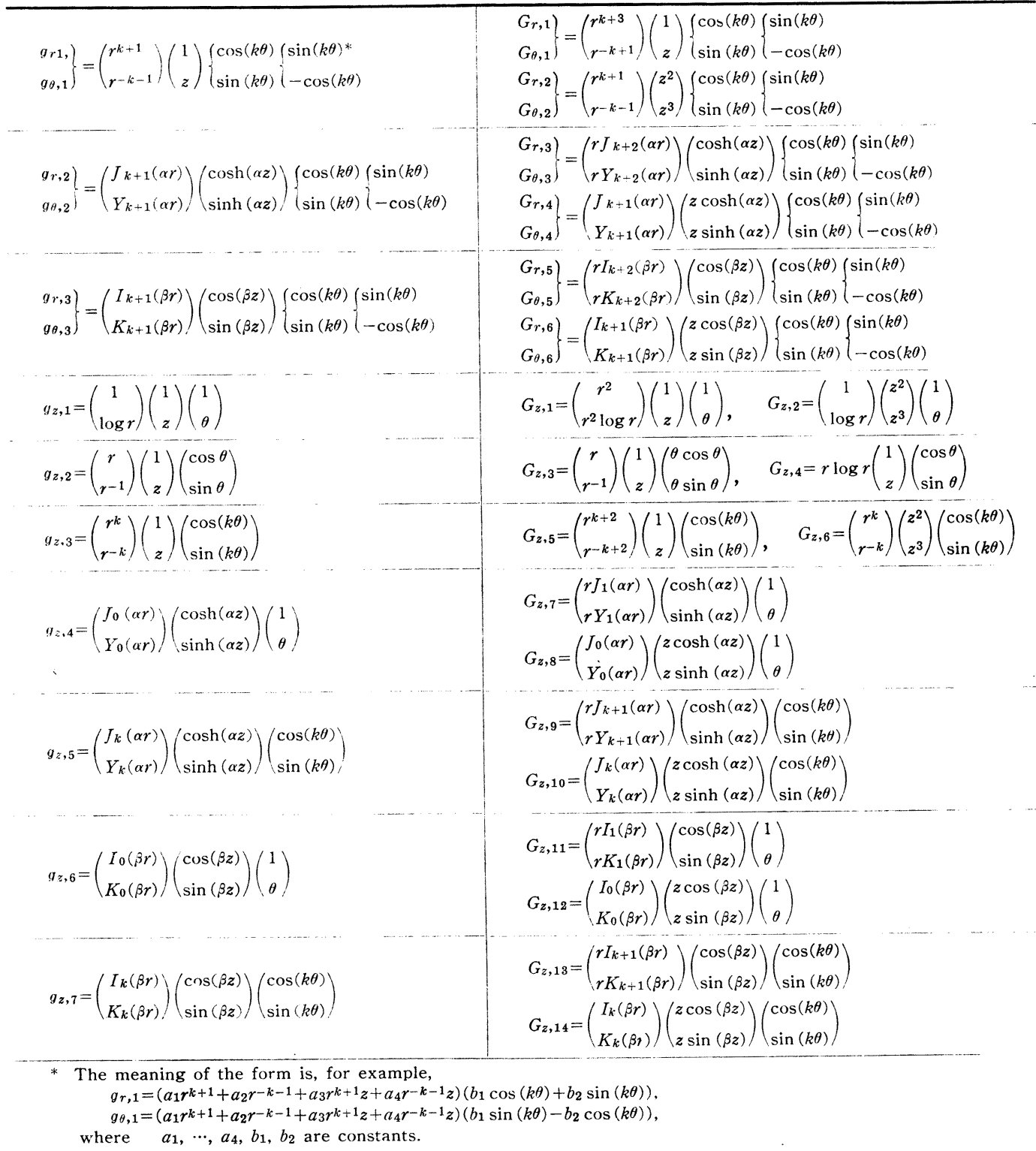

\section{EXAMPLE}

Okumura $^{8)}$ treated with the problem of an elastic short circular cylinder loaded semicircularly on its upper and lower sides by using the generalized Neuber's solution. We shall deal with the problem of an elastic short circular cylinder subjected to asymmetric loads (Fig. 1) as an application of the general forms of Galerkin vector explained in the previous chapter.
The boundary conditions are to be specified as follows:

$$
\begin{aligned}
& \text { on } r=a \\
& \tau_{r z}=0, \\
& \tau_{r \theta}=0, \\
& \sigma_{r}=0, \\
& \text { on } z= \pm h \\
& \tau_{r z}=0, \\
& \tau_{\theta z}=0,
\end{aligned}
$$




$$
\sigma_{z}=\left\{\begin{array}{c}
-\frac{p}{d} r \cos \theta \\
(0 \leqq r \leqq d) \\
0 \\
(d<r \leqq a)
\end{array}\right.
$$

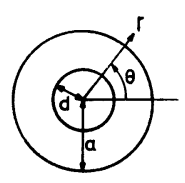

(16)

As the distributed loads are symmetrical for $z=0$ and proportional to $\cos \theta$, we choose the forms of Galerkin vector in the following manner:

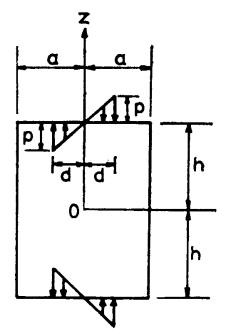

Fig. 1 Cylinder subjected to asymmetric loads.

$$
\begin{aligned}
& \boldsymbol{G}_{1}=A\left(\begin{array}{c}
r^{4} \cos \theta \\
r^{4} \sin \theta \\
0
\end{array}\right) \text {, } \\
& \boldsymbol{G}_{2}=\sum_{n=1}^{\infty} B_{n}\left(\begin{array}{c}
\frac{1}{2} r I_{3}\left(\beta_{n} \gamma\right) \cos \left(\beta_{n} z\right) \cos \theta \\
\frac{1}{2} r I_{3}\left(\beta_{n} \gamma\right) \cos \left(\beta_{n} z\right) \sin \theta \\
0
\end{array}\right) \text {, } \\
& \boldsymbol{G}_{3}=\sum_{m=1}^{\infty} C_{m}\left(\begin{array}{c}
0 \\
0 \\
-\frac{1}{\alpha_{m}} J_{1}\left(\alpha_{m} \gamma\right) \sinh \left(\alpha_{m} z\right) \cos \theta
\end{array}\right) \text {, } \\
& \boldsymbol{G}_{4}=\sum_{m=1}^{m} D_{m}\left(\begin{array}{c}
0 \\
0 \\
\frac{1}{2} J_{1}\left(\alpha_{m} \gamma\right) z \cosh \left(\alpha_{m} z\right) \cos \theta
\end{array}\right) \text {, } \\
& \boldsymbol{G}_{5}=\sum_{n=1}^{\infty} E_{n}\left(\begin{array}{c}
0 \\
0 \\
-\frac{1}{\beta_{n}} I_{1}\left(\beta_{n} r\right) \sin \left(\beta_{n} z\right) \cos \theta
\end{array}\right) \text {, } \\
& \boldsymbol{G}_{6}=\sum_{n=1}^{\infty} F_{n}\left(\begin{array}{c}
0 \\
0 \\
\frac{r}{2} I_{2}\left(\beta_{n} \gamma\right) \sin \left(\beta_{n} z\right) \cos \theta
\end{array}\right) \text {, }
\end{aligned}
$$

where $A, B_{n}, C_{m}, D_{m}, E_{n}$ and $F_{n}$ are arbitrary constants, and $\alpha_{m}$ and $\beta_{n}$ are given by the following equations respectively:

$$
\begin{aligned}
& \alpha_{m} J_{0}\left(\alpha_{m} a\right)=J_{1}\left(\alpha_{m} a\right) / a, \quad(m=1,2, \cdots), \\
& \beta_{n}=n \pi / h, \quad(n=1,2, \cdots) .
\end{aligned}
$$

Substituting Eq. (17) into Eq. (2), expressions of displacements are obtained. Using the relations between displacements and stresses, expressions of stresses are obtained. From the condi- tions of Eqs. (11) and (14), we obtain

$$
\begin{aligned}
& C_{m}=\left\{\nu+\alpha_{m} h /\left(2 \tanh \left(\alpha_{m} h\right)\right)\right\} D_{m}, \\
& E_{n}=L_{1} B_{n}+L_{2} F_{n}, \ldots \ldots \ldots \ldots \ldots \ldots \ldots \ldots \ldots \ldots \ldots
\end{aligned}
$$

where

$$
\begin{aligned}
L_{1}= & {\left[(\nu-3 / 2) \beta_{n} I_{0}\left(\beta_{n} a\right)+\{(3-2 \nu) / a\right.} \\
& \left.\left.+a \beta_{n}^{2} / 2\right\} I_{1}\left(\beta_{n} a\right)\right] /\left\{\beta_{n} I_{0}\left(\beta_{n} a\right)\right. \\
& \left.-I_{1}\left(\beta_{n} a\right) / a\right\} \\
L_{2}= & \left\{(1 / 2-\nu) \beta_{n} I_{0}\left(\beta_{n} a\right)+(\nu / a\right. \\
& \left.\left.+a \beta_{n}^{2} / 2\right) I_{1}\left(\beta_{n} a\right)\right\} /\left\{\beta_{n} I_{0}\left(\beta_{n} a\right)\right. \\
& \left.-I_{1}\left(\beta_{n} a\right) / a\right\} .
\end{aligned}
$$

From the conditions of Eqs. (12) and (13), we have

$$
\begin{aligned}
& \sum_{m=1}^{\infty} M_{1} D_{m}+M_{2} B_{n}+M_{3} F_{n}=0, \\
& \sum_{m=1}^{\infty} M_{4} D_{m}+M_{5} B_{n}+M_{6} F_{n}=0, \cdots \cdots \cdots \cdot \cdots \cdot(23) \\
& v / a^{2} h \sum_{m=1}^{\infty} D_{m} J_{1}\left(\alpha_{m} a\right) \sinh \left(\alpha_{m} h\right) / \alpha_{m}+12 a A=0,
\end{aligned}
$$

where

$$
\begin{aligned}
& M_{1}=2(-1)^{n} \alpha_{m} h J_{1}\left(\alpha_{m} a\right) \sinh \left(\alpha_{m} h\right) \\
& \cdot\left\{(\nu-1)(n \pi)^{2}+\nu\left(\alpha_{m} h\right)^{2}\right\} /\left[a ^ { 2 } \left\{\left(\alpha_{m} h\right)^{2}\right.\right. \\
& \left.\left.+(n \pi)^{2}\right\}^{2}\right] \text {, } \\
& M_{2}=\left[(3 \nu-7 / 2) \beta n^{2} / a I_{0}{ }^{2}\left(\beta_{n} a\right)+\left\{(9-8 \nu) / a^{2}\right.\right. \\
& \left.+(1-v) \beta_{n^{2}}\right\} I_{0}\left(\beta_{n} a\right) I_{1}\left(\beta_{n} a\right) \\
& \left.+\left\{4(1-\nu) / a^{3}+(\nu-1 / 2) \beta_{n}^{2} / a\right\} I_{1}^{2}\left(\beta_{n} a\right)\right] \\
& /\left\{\beta_{n} I_{0}\left(\beta_{n} a\right)-I_{1}\left(\beta_{n} a\right) / a\right\} \text {, } \\
& M_{3}=\left[(\nu-3 / 2) \beta_{n^{2}} / a I_{0}^{2}\left(\beta_{n} a\right)\right. \\
& +(4-3 v) \beta_{n} / a^{2} I_{0}\left(\beta_{n} a\right) I_{1}\left(\beta_{n} a\right) \\
& \left.+\left\{2(\nu-1) / a^{3}+\beta_{n^{2}} /(2 a)\right\} I_{1}^{2}\left(\beta_{n} a\right)\right] \\
& \mid\left\{\beta_{n} I_{0}\left(\beta_{n} a\right)-I_{1}\left(\beta_{n} a\right) / a\right\} \text {, } \\
& M_{4}=2(-1)^{n} \alpha_{m} h J_{1}\left(\alpha_{m} a\right) \sinh \left(\alpha_{m} h\right) \\
& \cdot\left\{(\nu-1)(n \pi)^{2}+\nu\left(\alpha_{m} h\right)^{2}+\left(a \alpha_{m} n \pi\right)^{2}\right\} \\
& /\left[a^{2}\left\{\left(\alpha_{m} h\right)^{2}+(n \pi)^{2}\right\}^{2}\right] \text {, } \\
& M_{5}=\left[\left\{(3 \nu-7 / 2) / a-a \beta n^{2} / 2\right\} I_{0}^{2}\left(\beta_{n} a\right)\right. \\
& +\beta_{n}\left\{\beta_{n}^{2}+(9-8 v) / a^{2}\right\} I_{0}\left(\beta_{n} a\right) I_{1}\left(\beta_{n} a\right) \\
& +\left\{4(\nu-1) / a^{3}+\beta_{n}^{2}\left((3 / 2-\nu) / a+a \beta_{n}^{2} / 2\right)\right\} \\
& \text { - } \left.I_{1}{ }^{2}\left(\beta_{n} a\right)\right] /\left\{\beta_{n} I_{0}\left(\beta_{n} a\right)-I_{1}\left(\beta_{n} a\right) / a\right\} \text {, } \\
& M_{6}=\left[\left\{(\nu-3 / 2) / a-a \beta n^{2} / 2\right\} I_{0}^{2}\left(\beta_{n} a\right)\right. \\
& +\beta_{n}\left\{\beta_{n}^{2}+(4-3 v) / a^{2}\right\} I_{0}\left(\beta_{n} a\right) I_{1}\left(\beta_{n} a\right) \\
& +\left\{2(\nu-1) / a^{3}+\beta n^{2}\left(1 / a+a \beta n^{2}\right) / 2\right\} \\
& \text { - } \left.I_{1}^{2}\left(\beta_{n} a\right)\right] /\left\{\beta_{n} I_{0}\left(\beta_{n} a\right)-I_{1}\left(\beta_{n} a\right) / a\right\}
\end{aligned}
$$

From the condition of Eq. (16), we get

$$
\sum_{l=1}^{\infty} N_{l} D_{l}+\sum_{n=1}^{\infty}\left(S_{1} B_{n}+S_{2} F_{n}\right)=U,
$$


where

$$
\begin{aligned}
N_{l}= & \begin{cases}{\left[\alpha_{m}^{2} / 2\left\{\left(1+\alpha_{m} h / \tanh \left(\alpha_{m} h\right)\right)\right.\right.} \\
\left./ \tanh \left(\alpha_{m} h\right)-\alpha_{m} h\right\} \\
\left.-8 v^{2} /\left\{\alpha_{m} a^{2} h\left(\left(\alpha_{m} a\right)^{2}-1\right)\right\}\right] \sinh \left(\alpha_{m} h\right), \\
-8 v^{2} J_{1}\left(\alpha_{l} a\right) \sinh \left(\alpha_{l} h\right) \\
/\left[\alpha^{2} h \alpha_{l}\left\{\left(\alpha_{m} a\right)^{2}-1\right\} J_{1}\left(\alpha_{m} a\right)\right], \quad l \neq m\end{cases} \\
S_{1}= & {\left[\beta_{n} T_{1} / 2+\left\{\beta_{n} I_{0}\left(\beta_{n} a\right) / 2-((2-v) / a\right.\right.} \\
& \left.\left.+\beta_{n}^{2} a / 2\right) I_{1}\left(\beta_{n} a\right)\right\} T_{2} /\left\{\beta_{n} I_{0}\left(\beta_{n} a\right)\right. \\
& \left.\left.-I_{1}\left(\beta_{n} a\right) / a\right\}\right](-1)^{n} \beta_{n}^{2} I_{1}\left(\beta_{n} a\right), \\
S_{2}= & {\left[\beta_{n} T_{1} / 2+\left\{\beta_{n} I_{0}\left(\beta_{n} a\right) / 2-(1 / a\right.\right.} \\
& \left.\left.+\beta_{n^{2}} a / 2\right) I_{1}\left(\beta_{n} a\right)\right\} T_{2} /\left\{\beta_{n} I_{0}\left(\beta_{n} a\right)\right. \\
& \left.\left.-I_{1}\left(\beta_{n} a\right) / a\right\}\right](-1)^{n} \beta_{n}^{2} I_{1}\left(\beta_{n} a\right), \\
T_{1}= & 2 \alpha_{m}^{2}\left[a^{2} \beta_{n}-\left\{a\left(\beta_{n}^{2}-\alpha_{m}^{2}\right) I_{0}\left(\beta_{n} a\right) I_{1}\left(\beta_{n} a\right)\right.\right. \\
& \left.\left.-\beta_{n}\left(1+a \alpha_{m} J_{0}\left(\alpha_{m} a\right) / J_{1}\left(\alpha_{m} a\right)\right)\right\} /\left(\alpha_{m}^{2}+\beta_{n}^{2}\right)\right] \\
& /\left[\left\{\left(\alpha_{m} a\right)^{2}-1\right\} J_{1}\left(\alpha_{m} a\right)\left(\alpha_{m}^{2}+\beta_{n}^{2}\right)\right], \\
T_{2}= & 2 a \alpha_{m}^{2}\left\{\beta_{n} I_{0}\left(\beta_{n} a\right) / I_{1}\left(\beta_{n} a\right)-1 / a\right\} \\
& /\left[\left\{\left(\alpha_{m} a\right)^{2}-1\right\} J_{1}\left(\alpha_{m} a\right)\left(\alpha_{m}^{2}+\beta_{n}^{2}\right)\right], \\
U= & 2 p\left\{\alpha_{m} d J_{0}\left(\alpha_{m} d\right)-2 J_{1}\left(\alpha_{m} d\right)\right\} \\
& /\left[\left\{\left(\alpha_{m} a\right)^{2}-1\right\}\left\{J_{1}\left(\alpha_{m} a\right)\right\}^{2}\right] .
\end{aligned}
$$

The condition of Eq. (15) is satisfied identically by Eqs. (19) and (20). From Eqs. (23), (24), (25) and (27), we obtain the simultaneous equations for $A, B_{n}, D_{m}$ and $F_{n}$. The calculations are carried out numerically, in which the first $m_{0}$

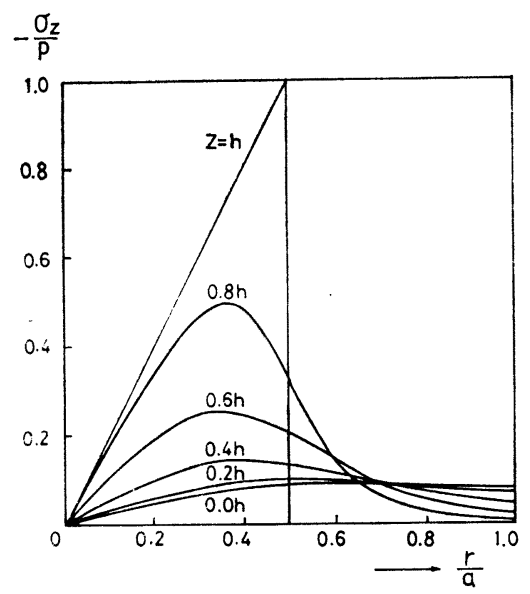

Fig. 2 Stress distribution of $\sigma_{z}$ at $\theta=0$.

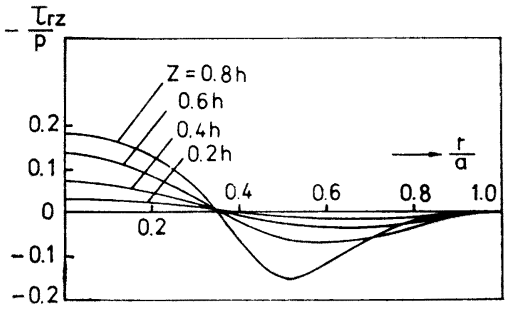

Fig. 3 Stress distribution of $\tau_{r z}$ at $\theta=0$.

roots of $\alpha_{m}$ and $n_{0}$ roots of $\beta_{n}$ are used. Assuming the following values

$$
d / a=0.5, \quad a / h=1.0, \quad \nu=0.25,
$$

we get the results illustrated in Figs. 2 and 3 , which have 3 digits of efficient numbers, taking $m_{0}=n_{0}=15$.

\section{CONCLUDING REMARKS}

In this paper, the solution of Navier's equation represented by Galerkin vector was investigated in cylindrical coordinates. General forms of Galerkin vector in cylindrical coordinates were listed in Table 1 and the proposed forms of Galerkin vector were applied efficiently to an asymmetric problem in cylindrical coordinates.

\section{REFERENCES}

1) Y. C. Fung: Foundation of Solid Mechanics, Prentice Hall, p. 184, 1965.

2) J. Boussinesq: Applications des Poténtiels, Gauthier-Villars, 1885.

3) A. E. H. Love: A treatise on the mathematical theory of elasticity, Dover, p. 274, 1944

4) H. Hasegawa: Trans. Japan Soc. Mech. Engrs., vol. 40 , p. 2497, 1974.

5) R. Muki: Proc. 5th Japan Natl. Congr. Appl. Mech., p. 38, 1955.

6) K. Hata: Bull. Fac. Eng., Hokkaido Univ., vol. 13 , p. 14, 1955.

7) Y .C. Fung: op. cit. p. 207.

8) I. Okumura: Theoretical and applied mechanics, vol. 24, p. 249, 1974.

(Received December 19, 1978) 